\title{
TREATMENT OF VITILIGO WITH AUTOLOGOUS EPIDERMAL TRANSPLANTATION USING THE ROOF OF SUCTION BLISTERS, FIRST IN BANGLADESH
}

\author{
KHUNDKAR $\mathrm{SH}^{1}$, RABBI SMR ${ }^{2}$, AWWAL R $^{3}$, HUSSAIN DAS $^{4}$
}

\begin{abstract}
We report our experience of autologous epidermal transplantation for 30 (Thirty) patients with vitiligo. The vitiligo in 25 (Twenty five) patients was stable whereas in the rest (5) it was active. Autologous epidermal transplantation was performed using suction blister roofs from normal pigmented skin to vitiligo skin that was failed to repigment using topical steroid and/or psoralenultraviolet- A treatment. Grafts were well taken in all the patients. Only three of them are presented as case report in this article. There were no complications except mild hyper pigmentation at the donor areas. For the patient who had active vitiligo, depigmentation of the graft and concomitant Koebner's phenomenon at the donor site were observed 3 weeks after the procedure. We conclude that autologous epidermal transplantation using the roof of suction blisters is an excellent and safe regimenting procedure for stable, localized vitiligo and the active disease precludes transplantation.
\end{abstract}

Key word: Epidermis surgery, vitiligo therapy, transplantation alutologous.

J Dhaka Med Coll. 2009; 18(1) : 58-63

\section{Introduction :}

Vitiligo is a worldwide acquired disease of unknown aetiology and characterized by spontaneous skin depigmentation due to loss of melanocytes. The disease has prevalence of $1 \%$ to $2 \%$ and up to $8.8 \%$ in some countries. Most afflicted patients enjoy good general health; however, the disease can have a profound psychological impact, which may lead to a disturbed social life. Conventional therapies include the use of topical and/or systemic steroid, and psoralen-ultraviolet $\mathrm{A}$ (PUVA) treatment. These treatments achieve varying degrees of repigmentation and their response may be appreciated only after a long period of frequent and regular treatment- In addition, the necessity of using prolonged treatment increases the chance of exposure to the adverse effects of these agents Various surgical methods that aim to replenish lost melanocytes have gained popularity in recent years. Among them is autologus epidermal transplantation (AET) using suction blisters, which appears to be very promising for treating stable, localized vitiligo. We report our experience of AET using the roof of suction blisters in thirty patients: twenty-five with stable localized vitiligo lesions and five with active vitiligo. The technique of the procedure is also described in this article.

\section{Epidermal Transplantation Technique}

The donor skin at the medial aspect of the right/left thigh was disinfected, as in any surgical procedure. Glass cups (or syringes) of appropriated artificial size were placed on the donor skin and suction was applied at a constant negative pressure. The pressure ranged from -33.3 to $-40.0 \mathrm{kpa}$ and was created by an SAM12 electric vacuum suction (MG Medical, Colchester, UK). No anesthesia was required because the procedure is painless. Small vesicles started to form after about 1 hour of continuous suction, which enlarged and

1. Department of Plastic and Reconstructive Surgery, Dhaka Medical College and Hospital

2. Medical officer, Laser Medical Center, Dhanmondi, Dhaka.

3. Assistant Professor, Department of Plastic and Reconstructive Surgery, Dhaka Medical College and Hospita, Dhaka

4. Assistant Professor, Department of Biochemistry(Moulana Bhasani Medical College), Dhaka

Correspondence: Prof. Shafquat Hossain Khundkar. 
coalesced to form a bulla approximately 1 hour later. The roof of the bulla, which represented the epidermal graft, was separated from the surrounding skin using a pair of curved iris scissors. The graft, supported by moistened plain fine gauze, was placed on a platform and a pair of fine forceps was used to gently spread it to its maximum size. Any fibrin clots attached to the dermal surface were gently removed. The graft was trimmed to a size and shape appropriate to the recipient site (vitiliginous skin), which had been prepared simultaneously in the same way as the donor area or by derma abrasion. The graft was accurately implanted into the recipient site. The procedure was completed after applying antibiotic spray and covering the graft with paraffin gauze, then plain gauze, and finally semipermeable adhesive dressing.

\section{Case Reports}

\section{Case 1:}

A 24-year-old female student was referred by a skin specialist from Chittagong 2008. A white patch appeared spontaneously on the left side of the patient's chin about 6 month before the consultation. The lesion started as a small white spot and enlarged within a few months. On examination, a well-demarcated, depigmented triangular patch $2.0 \mathrm{~cm} \mathrm{X} 3.0 \mathrm{~cm}$ $X 4.0 \mathrm{~cm}$ was found abutting the lower lip on the left side of the chin. Wood's lamp illumination accentuated the depigmentation and supported a diagnosis of vitiligo. There was no vitiliginous lesion elsewhere on the face, body, or limbs. The patient was treated with topical $0.03 \%$ tacrolimus cream and $1 \%$ Pimecrolimus cream (Novartis) for one year, with no response. Then topical PUVA treatment was given twice weekly for 6 months. The therapy was temporarily suspended due to a PUVA induced burn; thereafter, treatment continued uneventfully. Therapy was stopped after another 6 months due to lack of repigmentation. Since the consultation, the size of the vitiliginous patch had remained unchanged. AET using the suction blister method was advised and preoperative evaluation including complete blood count, renal and liver functions, thyroid stimulating hormone and total thyroxine was done. All tests were normal or negative. Autologous epidermal transplantation was performed in Laser Medical Center on $5^{\text {th }}$ June 2008; epidermal graft was taken from the medial aspect of the patient's thigh. Observation after removing the dressing on day 14 th revealed a very good uptake of the graft. Faint uneven pigmentation was noted on the recipient site after the epidermal graft had sloughed off a few days later. Subsequent follow-up showed that pigmentation had spread and intensified. Ultimately an evenly pigmented patch was formed within two months after grafting.

\section{Case 2:}

A 17 years old female from Dhaka had depigmented patches that appeared spontaneously on the right side of his neck for 5 years. The patient's skin was otherwise normal. He enjoyed good general health and had no family history of vitiligo or autoimmune diseases. Under Wood's lamp illumination, the patches appeared white, thus confirming a clinical diagnosis of vitiligo. Systemic examination was normal. The same blood tests were done as for case 1 and results were also normal or negative.

The patient was treated with Tacrolimus cream for 5 months. But no improvement was noted. She refused topical PUVA treatment as it takes a long time. Autologous epidermal transplantation was offered as treatment option and accepted. The grafting method used was the same as for Case 1. A total of three epidermal sheets $(2.2 \mathrm{~cm} \mathrm{X} 1.5 \mathrm{~cm}, 1.2 \mathrm{~cm} \mathrm{X}$ $0.9 \mathrm{~cm}$, and $1.4 \mathrm{~cm} \mathrm{X} 1.0 \mathrm{~cm}$ ) were grafted. Follow up at an interval of one and three months after the procedure showed satisfactory repigmentattion in the grafted areas. A second AET for the rest of the vitiliginous lesions was suggested but refused for lack of time and also because a satisfactory cosmetic result had already been achieved.

\section{Case 3:}

A 37 years old female purchaser first noticed white patches on the right zygomatic area and angles of the mouth in 2008. Further white patches appeared on the right lower neck, 
abdomen, and distal fingers. The patient was otherwise healthy and had no family history of vitiligo, thyroid, or other autoimmune diseases. Prior treatments with topical steroids had temporary improvement in some lesions. New lesions also appeared during treatment period.

The patient was advised for PUVA treatment and no apparent improvement was noticed after approximately 8 months of therapy and then she was referred for AET. Her history strongly suggested that she had active vitiligo and thus contra-indicated for any surgical repigmentation procedure. The results of blood tests and thyroid function however were

1 (a)

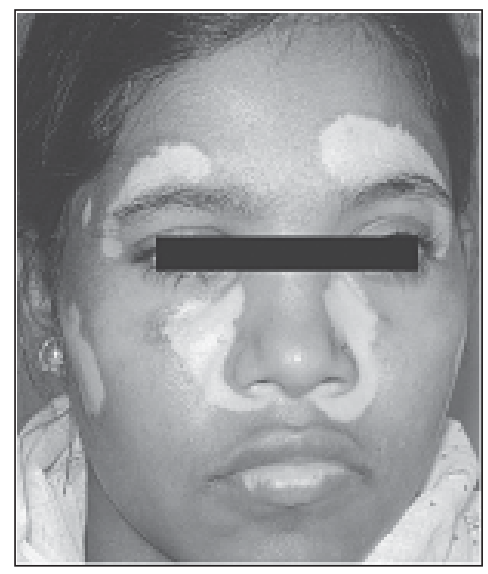

2 (a)

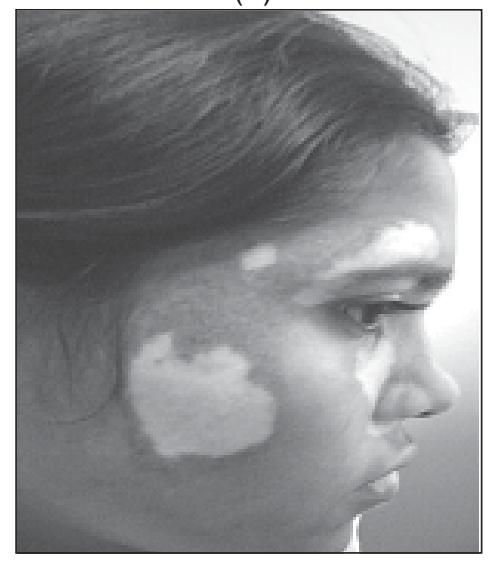

Before operation normal or negative. At the patient's strong request and acceptance of possible complications, a single epidermal sheet of 1.8 $\mathrm{cm}$ diameter was taken from a normally pigmented area on the right side of the thigh and was grafted on the lesion of her right lower neck. A follow up of 8 days after the procedure revealed very good graft uptake. Three weeks after the procedure the size of the pigmented area was notably reduced. However, a faint depigmentation was noted at the donor site of the thigh which is a feature of Koebner's phenomenon. The patient's request for further transplantation was rejected.

1 (b)

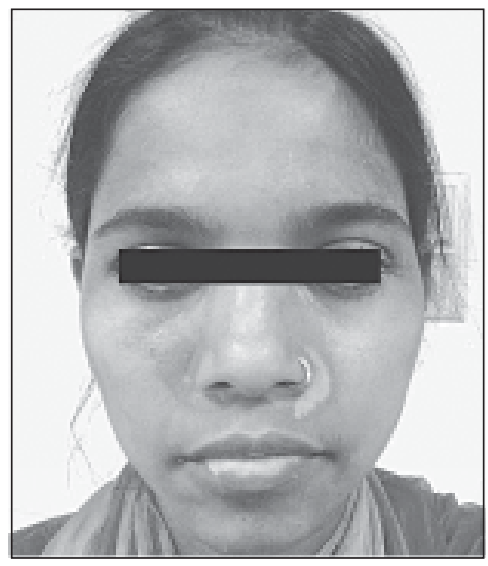

2 (b)

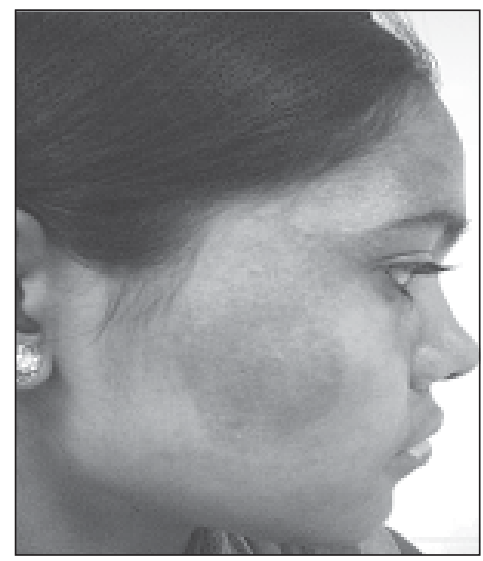

After operation

(Autologous epidermal transplantation)

Fig.-1: Photographs of Vitiligo lesion before and after treatment

(la) Vitiliginous patch of the face. (lb) Follow-up six months after the first treatment revealed very satisfactory pigmentation at the first grafted area. Re-pigmentation continued at the more recently grafted areas. 


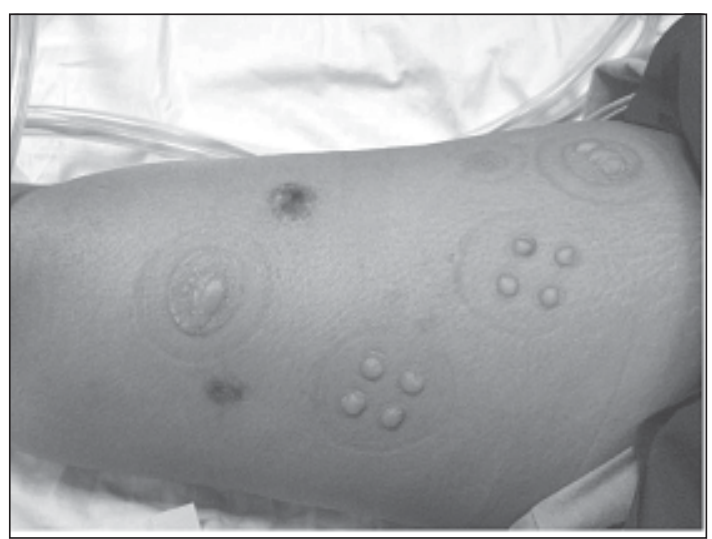

(a) Photograph showing suction blister at donor site of thigh.

bollas of a size comparable to the orificial size of the suction cup formed at the donor area after one hours continuous suction.

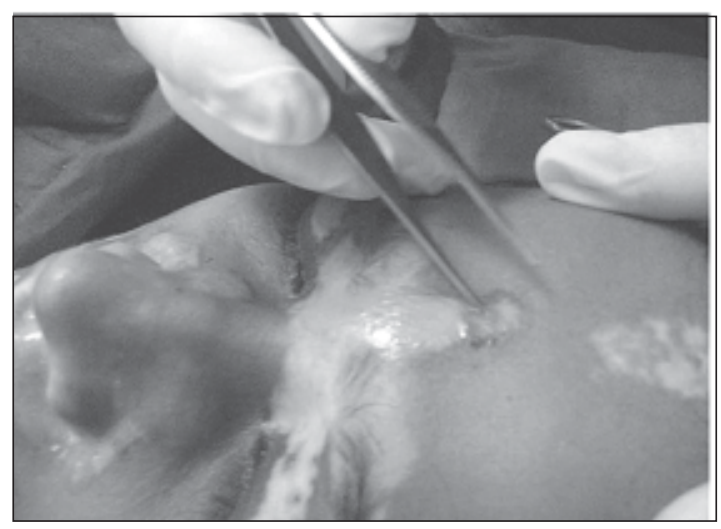

(c) Blister replaced on the recipient area.

\section{Discussion}

Vitiligo is often regarded by physicians as a disease not readily amenable to treatment and by the general public as incurable. Transplantation of melanocytes is an alternative and effective way to repigment stable vitiligo that has failed steroid and/or PUVA treatment. The principle of melanocyte transplantation is simple and involves the harvesting of melanocytes (donor graft), removal of the vitiliginous skin or epidermis (recipient area) followed by implantation of the donor graft into the prepared recipient area. The harvesting of melanocytes can be accomplished by the creation of a donor graft using surgical methods or by the in vitro culture of melanocytes using special growth

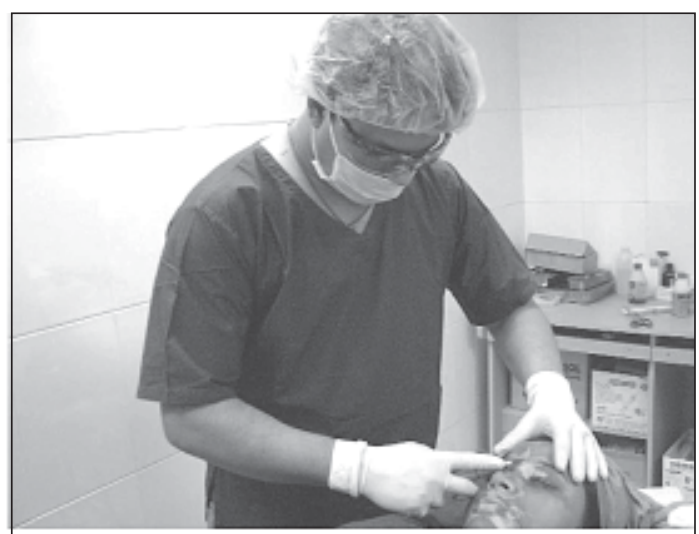

(b) Photograph showing preparation of the recipient site (Vitiligo area) Abrasion of Vitiligo area by using derma abrasion.

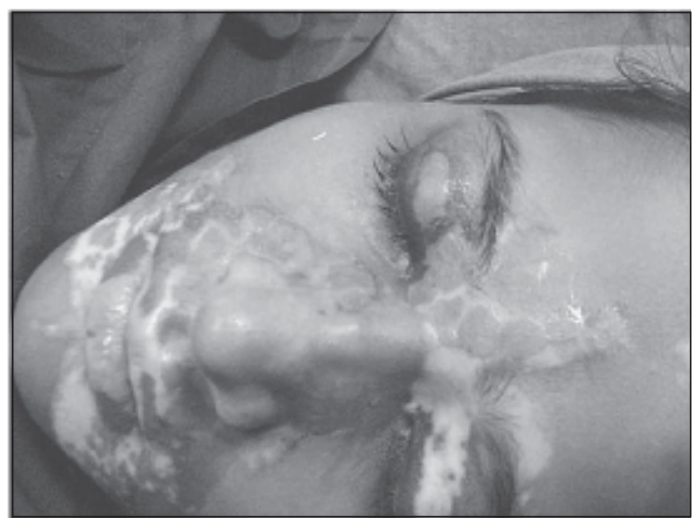

(d) Photograph showing autologous epidermal transplantation

media. Surgical methods for harvesting normally pigmented donor skin have been practiced for many years. This include the use of Thiersch's split thickness skin graft and mini punch graft. All produce good results of pigmentation. These methods, however, have not gained wide popularity because they require anesthesia. In addition, the surgery carries a risk of damage to the dermis and hence scarring.

In contrast, AET using roofs of suction blisters is an effective, non scarring \& minimally invasive surgical repigmentation method that has become popular since 1971, when Falabella reported his success in treating four patients with leukoderma. The AET repigmenting procedure is based on the principle discovered 
by kiistala and Mustakallion in 1964. The prolonged suction of the skin in vivo can produce dermo epidermal separation which results in the formation of a blister. The roof of the blister comprises the whole epidermis, which includes an intact basal cell layer where most melanocytes and melanin reside, and can therefore be used as a donor graft in AET for treating vitiligo. That the immediately underlying dermis is intact explains why there is no procedural pain and why epidermal regeneration is possible without scar formation. The procedure is therefore repeatable. The results from centers that have used this method for AET are most remarkable in patients with segmental vitiligo especially on the face. Incidentally, the epidermis obtained by this method has also been utilized for treating exposed dermal surfaces of patients with burns.

The patient described in Case 1, the first vitiligo patient in our service in Bangladesh treated with AET using the roofs of suction blister, had sparse pigmentation with uneven density at 1 month follow up. The density later intensified and simultaneously had spread towards the periphery of the original recipient area. Our observation from this study suggests that maximum pigmentation occurred within the first 2 to 3 months; thereafter, naked eye changes were subtle. No visually discernible difference could be noted between follow up at sixth and ninth month after AET. The repigmenation process in this patient was also recorded by measuring the total area of pigmentation once every 1 to 2 months over a period of 9 months, using small, regular $2 \mathrm{~mm}$ lattice squares. Such measurements of repigmented area, at the end of one month and six months after procedure, were $112 \mathrm{~mm}^{2}$ and $164 \mathrm{~mm}^{2}$ respectively.

The proliferation and in vitro migration of melanocytes account for the increase in the size of the pigmentation and the final even distribution of pigmentation. Transfer of melanosomes from melanocytic dendrites to the neighbouring keratinocytes within an epidermal-melanin unit is also a contributing factor. The Maximum area of pigmentation $\left(164 \mathrm{~mm}^{2}\right)$ was nevertheless smaller than the original donor graft $\left(209 \mathrm{~mm}^{2}\right)$ and this partly contributed to the formation of an achromatic fissure. We therefore propose that a donor graft obtained from the roof of a suction blister should not be smaller than the corresponding recipient area and that prior accurate measurement of recipient sites can help to reduce the incidence of achromatic fissures and hence achieve a more superior cosmesis. Equally good repigmentation was seen in case 2 at the last follow up of 9 months after AET. Case 3 had an ongoing active disease and failed to sustain satisfactory repigmentation. The creation of a blister also induced Koebner's phenomenon in this patient's donor area which resulted in depigmentation.

Based on our experience with these three patients, we conclude that stable vitiliginous lesions are amenable to AET. On the other hand, patient with ongoing active vitiligo, as the patient described in case 3 are not suitable for AET until their disease has become inactive. Furthermore, for AET to be performed, it should be ensured that Koebner's phenomenon does not occur.

In three patients of this study, lesions were located on the chin and neck areas and blisters were obtained quite easily using negative pressure suction from thigh. Post-operative graft immobilisation was also not difficult. At present, ideal candidates of vitiligo are treated by AET in our clinic and their results so far appear good. Practical problems from the AET procedure have occurred but are uncommon. These include the necessity for prolonged suction for blister formation (at least 2 hours), thus precluding the application of this procedure for young children; location of recipient sites in joints and uneven areas which hinder the secure application of suction cups; hairy and tight recipient skin which impedes blister formation; and premature detachment of dressing and grafts. With refinement of our skill, utilization of modern technology and with continuous patients request for $\mathrm{AET}$, we are optimistic that these problems can be solved. 
Table-I

Post operative evalution of patients( $N=30)$

\begin{tabular}{lcc}
\hline Result & No. of patient & $\begin{array}{c}\text { Percentage to melanocyte } \\
\text { cell come }\end{array}$ \\
\hline $\begin{array}{l}\text { Satisfactory } \\
\text { Excellent (16) } \\
\text { Good (9) }\end{array}$ & 25 (Stable) \\
$\begin{array}{l}\text { Unsatisfactory } \\
\text { Fair (3) } \\
\text { Poor (2) }\end{array}$ & 5 (Unstable / Active) \\
\hline
\end{tabular}

\section{References:}

1. Mosher DB, Fitzpatrick TB, Hori Y, Ortonne JP, Disorders of pigmeatation. In : Fitzpatrick AZ, Wolff K, Freedberg IM, Austen KF (editors), Dermatology in general Mediciane, New York: Mc Graw Hill, 1993; 01: 903-95.

2. Porter J, Beef AH, Lerner A, Nordlund J. Response to cosmetic disfigurement: patients with vitiligo. Cutis Int J Dermatol. 1987; 39: 493-4.

3. Nordlund JJ, Halder RM, Grimes P. Management of vitiligo. Dermatol Clin, 1993; 01: 27-33.

4. Harrist TJ, Pathak MA, Mosher DB, Fitzpatrick TB. Chronic Cutaneous Effects of long-term Psoralen and ultraviolet radiation therapy in patients with vitiligo. Natl Cancer Inst Monogr. 1984; 66:191-6.

5. Behl PN, Bhatia RK. Treatment of vitiligo with autologous thin thiersch's grafts. Int $\mathrm{J}$ dermatol. 1973;12: 329-31

6. Agrawal K, Agrawal A. Vitiligo: repigmentation with demabrasion and thin split-thicknessskin graft. Dermatol Surg. 1995; 295-300.

7. Falabell R. Repigmentation of segmental vitiligo by autologous minigrafting. J Am Acad Dermatol. 1983; 12: 329-31.

8. Falabella R. Repigmentation of segmental Vitiligo by autologous minigrafting: an original technique and its application in achromic and granulating areas. Arch Dermatol. 1971; 104: 592-600.

9. Kiistala U, Mustakallio KK. In-vivo separation of epidermis by production of suction blister Lancers Lancet. Dermatol Surg. 1964; 1:144-5.

10. Hann SK, Bong HW, Park YK. Treatment of stable vitiligo with autologous epidermal and PUVA. J Am Acad Dermatol. 1995; 32: 943-8.

11. Heck EL, Bergstresser PR, Baxter CR. Composite skin graft: frozen dermal allografts support the engraftment and expansion of autologous epidemis. J Traum. 1985; 25: 106-12.

12. Jimbow K, QuevedoWC, Jr Fitzpatrick TB, Eisen AZ, Wolff K, Freedberg IM, Austen KF (editors). Dermatology in general medicine, New York: Mc Graw-Hill, 1993; 01: 261-89.

13. Hatchome N, Kato T, Tagmi H. Therapeutic success of epidermal grafting in generalized vitiligo is limited by the Koebner phenomenon. J Am Acad Dermatol. 1990; 22: 87-91.

14. Koga M. Vitiligo : a new classification and therapy. Br J Dermatol. 1977; 97: 255-61.

15. Hann SK, Lee HJ. Segmental Vitiligo: Clinical Findings in 208 patients. J Am Acad Dematol. 1996; 35: 671-4.

16. Grimes PE. Psoralen Photochemotherapy for vitiligo. Clin Dermatol. 1997; 15: 921-6. 